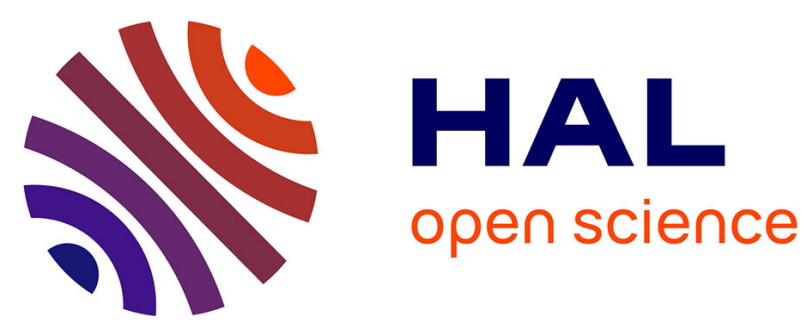

\title{
Why optical nonlinear characterization using imaging technique is a better choice?
}

\author{
Georges Boudebs, V. Besse, C. Cassagne, H. Leblond, F. Sanchez
}

\section{To cite this version:}

Georges Boudebs, V. Besse, C. Cassagne, H. Leblond, F. Sanchez. Why optical nonlinear characterization using imaging technique is a better choice?. 15th International Conference on Transparent Optical Networks (ICTON), Jun 2013, Cartagena, Colombia. pp.1-4, 10.1109/ICTON.2013.6602866 . hal-02442887

\section{HAL Id: hal-02442887 \\ https://hal.science/hal-02442887}

Submitted on 20 Jan 2020

HAL is a multi-disciplinary open access archive for the deposit and dissemination of scientific research documents, whether they are published or not. The documents may come from teaching and research institutions in France or abroad, or from public or private research centers.
L'archive ouverte pluridisciplinaire HAL, est destinée au dépôt et à la diffusion de documents scientifiques de niveau recherche, publiés ou non, émanant des établissements d'enseignement et de recherche français ou étrangers, des laboratoires publics ou privés. 


\title{
Why optical nonlinear characterization using imaging technique is a better choice?
}

\author{
G. Boudebs ${ }^{(*)}$, V. Besse, C. Cassagne, H. Leblond and F. Sanchez \\ LUNAM Université, Université d'Angers, LPhiA, Laboratoire de Photoniques d'Angers, EA 4464, 49045 Angers \\ Cedex 01, France \\ (*) Tel:(33) 2.41.73.54.26,Fax:(33) 2.41.73.52.16, email: georges.boudebs@univ-angers.fr
}

\begin{abstract}
We show that the direct measurement of the beam radius in Z-scan experiments using a CCD camera at the output of a 4 f-imaging system allows a higher sensitivity and a better accuracy than Baryscan. One of the advantages is to be insensitive to pointing instability of the pulsed laser because no hard aperture is employed as in the usual Z-scan. In addition, the numerical calculations involved here and the measurement of the beam radius are simplified since we do not measure the transmittance through an aperture and it is not subject to mathematical artifacts related to a normalization process, especially when the diffracted light is very low.
\end{abstract}

Keywords: nonlinear optics, Z-scan, diffraction, image processing, Fourier optics

\section{INTRODUCTION}

The inspection of linear and nonlinear (NL) optical parameters such as refractive index and absorption coefficient is essential for most of the applications in optics (optical limiting, lasers, optical amplifiers, photovoltaic cells, ablation, surgery, all-optical switching...). Measuring the NL refraction and absorption using the far field beam diffraction as in Z-scan method [1] is a widely used method nowadays. More recently a variant of this method called Baryscan [2] reporting an increased sensitivity has been published. The authors check the displacement of the beam centroid caused by the focusing (or defocusing) effect induced by the nonlinearity and achieved by a razor blade positioned close to a Position Sensitive Detector (PSD). The razor blade truncates half of the probe beam indicating an increase (or a decrease) of the beam size. Then the PSD shows a change in the position of the beam barycenter. The authors of Ref [2] claim sensitivity up to $\lambda / 50000$ using stable CW solidstate laser input. Recently van Stryland's group reported a new dual-arm Z-scan technique [3] improving the sensitivity up to $\lambda / 1000$ using pulsed lasers. This new method allowed the determination of NL refractive signals considerably smaller than usually found in the single-arm Z-scan method. These studies show that the sensitivity is still relevant and an active research field especially when it was understood that the normalization in the Z-scan method plays an important role. This problem in eclipsing experiments and its consequence in the normalisation process was pointed out first in Ref. [4]. Therefore, the consideration of the diffraction efficiency allowed to compare the sensitivity of different measurement techniques inside a Z-scan 4f-system [5] and had the precious advantage to avoid a "division by zero" with non existing energy. The advantage of using the diffraction efficiency allowed us to increase both the sensitivity of the measurement and the signal to noise ratio by matching properly the object with the field-stop. This optimization is not subject to artefacts linked to a normalization process when dividing by very low intensity profiles. It was shown that the Z-scan is the most sensitive techniques with the highest signal-to-noise ratio allowing measurements of NL induced phase distortions as small as $\lambda / 1000$. But still remain open questions to fully understand the physical phenomenon improving the sensitivity as claimed in [2]. The use of a two-dimensional CCD detector is a more accurate and easier method [6] and accordingly, Tsigaridas et al presented a modification of Z-scan technique by directly measuring the beam radius as a function of the peak irradiance [7].

We will show here that the use of hard-physical apertures (as razor blade) in the Baryscan technique under real pulsed laser with pointing fluctuations could be a severe limitation leading to both lower sensitivity and signal-to-noise ratio when compared to the direct measurement of the output beam waist variation using a CCD sensor.

\section{THE THEORETICAL MODEL}

The NL image formation inside the $4 f$ coherent imaging system (Fig. 1) can be described using a simple model based on Fourier optics (see for example Refs. [8], [9]). In this section we recall this approach. The general scheme of beam propagation inside the $4 f$ imaging system is described in details in [5].

Let us suppose that the amplitude of electric field distribution at the object plane is Gaussian, $E(x, y)=E_{0} \exp \left[-\left(x^{2}+y^{2}\right) / \omega_{e}^{2}\right]$, where $x, y$ are the spatial coordinates, $E_{0}$ denotes the on-axis amplitude and $\omega_{e}$ is the beam waist at the entry of the setup. Let $S(u, v)$ be the spatial spectrum of $E(x, y)$ : 
$\mathrm{S}(\mathrm{u}, \mathrm{v})=\tilde{\mathcal{F}}[\mathrm{E}(\mathrm{x}, \mathrm{y})]=\int_{-\infty}^{+\infty} \int_{-\infty}^{+\infty} \mathrm{E}(\mathrm{x}, \mathrm{y}) \exp [-\mathrm{j} 2 \pi(\mathrm{ux}+\mathrm{vy})] \mathrm{dxdy}$, where $\tilde{\mathcal{F}}$ denotes the Fourier transform operation, $\mathrm{u}$ and $\mathrm{v}$ are the normalized spatial frequencies.

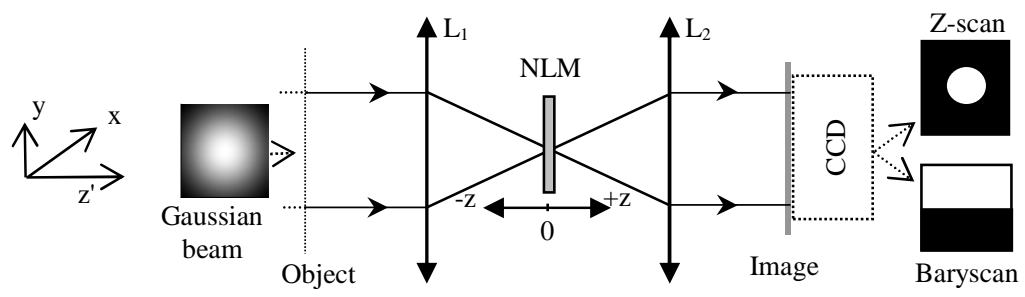

Figure 1: The 4 imaging system. The nonlinear material (NLM) is moved around the focal plane. The labels refer to: lenses $\left(L_{1}-L_{2}\right)$. The object at the entry is a Gaussian beam, while a circular aperture or a razor blade can be considered to as soft apertures spatially filtering the image to obtain Z-scan or Baryscan

The spectrum of the object is propagated over a distance $z^{\prime}$, by taking into account the transfer function of the wave propagation phenomenon $\mathrm{H}(\mathrm{u}, \mathrm{v})=\exp \left(\mathrm{j} 2 \pi \mathrm{z}^{\prime} \sqrt{1-(\lambda \mathrm{u})^{2}-(\lambda \mathrm{v})^{2}} / \lambda\right)$, where $\lambda$ is the wavelength. The field amplitude at $\mathrm{z}^{\prime}$ after free propagation is obtained by computing the inverse Fourier transform: $\mathrm{E}\left(\mathrm{x}, \mathrm{y}, \mathrm{z}^{\prime}\right)=\tilde{\mathcal{F}}^{-1}[\mathrm{~S}(\mathrm{u}, \mathrm{v}) \mathrm{H}(\mathrm{u}, \mathrm{v})]$. To calculate the output beam after passing through a lens with focal length $\mathrm{f}$, we apply the phase transformation related to their thickness variations in paraxial approximation: $\mathrm{t}_{\mathrm{L}}(\mathrm{x}, \mathrm{y})=\exp \left[-\mathrm{j} \pi\left(\mathrm{x}^{2}+\mathrm{y}^{2}\right) / \lambda \mathrm{f}\right]$. The first propagation is performed on a distance $\mathrm{z}^{\prime}=\mathrm{f}$ from the object plane to the lens $L_{1}$. Then we propagate the beam up to the sample located at $\mathrm{z}$ using $\mathrm{z}^{\prime}=\mathrm{f}+\mathrm{z}$ in $\mathrm{H}$ the optical transfer function $\left(z=0\right.$ at the focus of the lens $L_{1}$ ). After that the NL response of the material is taken into account. We assume cubic nonlinearity and a thin NL medium of thickness L exhibiting (i) linear absorption defined by $\alpha\left(\mathrm{m}^{-1}\right)$, (ii) two-photon absorption defined by $\beta(\mathrm{m} / \mathrm{W})$ and (iii) NL refraction defined by $\mathrm{n}_{2}\left(\mathrm{~m}^{2} / \mathrm{W}\right)$. In these conditions the transmittance of the sample is described as:

$$
\mathrm{T}(\mathrm{u}, \mathrm{v}, \mathrm{z})=\frac{\mathrm{S}_{\mathrm{L}}(\mathrm{u}, \mathrm{v}, \mathrm{z})}{\mathrm{S}(\mathrm{u}, \mathrm{v}, \mathrm{z})}=\left\{\mathrm{e}^{\alpha \mathrm{L}}[1+\mathrm{q}(\mathrm{u}, \mathrm{v}, \mathrm{z})]\right\}^{-1 / 2} \exp \left[\mathrm{j} \Delta \phi_{\mathrm{NL}}^{\mathrm{eff}}(\mathrm{u}, \mathrm{v}, \mathrm{z})\right]
$$

where $\mathrm{q}(\mathrm{u}, \mathrm{v}, \mathrm{z})=\beta \mathrm{L}_{\text {eff }} \mathrm{I}(\mathrm{u}, \mathrm{v}, \mathrm{z})$ with $\mathrm{L}_{\text {eff }}=\left(1-\mathrm{e}^{-\alpha \mathrm{L}}\right) / \alpha$ representing the effective length and $\mathrm{I}(\mathrm{u}, \mathrm{v}, \mathrm{z})$ denotes the intensity of the laser beam within the sample. The latter quantity is related to the input electric field through the expression $\mathrm{I}(\mathrm{u}, \mathrm{v}, \mathrm{z}) \propto|\mathrm{S}(\mathrm{u}, \mathrm{v}, \mathrm{z})|^{2}$. Here $\Delta \phi_{\mathrm{NL}}^{\text {eff }}(\mathrm{u}, \mathrm{v}, \mathrm{z})$ can be considered as the effective NL phase shift: $\Delta \phi_{\text {NL }}^{\text {eff }}(\mathrm{u}, \mathrm{v}, \mathrm{z})=2 \pi \mathrm{n}_{2} \mathrm{~L}_{\text {eff }} \mathrm{I}_{\text {eff }}(\mathrm{u}, \mathrm{v}, \mathrm{z}) / \lambda$ where $\mathrm{I}_{\text {eff }}(\mathrm{u}, \mathrm{v}, \mathrm{z})=\mathrm{I}(\mathrm{u}, \mathrm{v}, \mathrm{z}) \log [1+\mathrm{q}(\mathrm{u}, \mathrm{v}, \mathrm{z})] / \mathrm{q}(\mathrm{u}, \mathrm{v}, \mathrm{z})$ is the effective intensity seen by the material for Gaussian beams. Let $q_{0}=\beta L_{\text {eff }} I_{0}$ and $\varphi_{\mathrm{NL} o}^{\text {eff }}=\Delta \phi_{\mathrm{NL}}^{\text {eff }}(0,0,0)$ denote the on-axis NL absorption and NL phase shift at the focus, respectively, $\mathrm{I}_{0}$ is the focal on-axis intensity.

Next, the measurement of the beam waist can be performed using the ISO standard definition. Based on the second moment of $\mathrm{I}(\mathrm{x}, \mathrm{y})$ the D4 $\sigma$ method gives 4 times the standard deviation of the intensity distribution. For example, the beam radius in the $\mathrm{x}$ direction is:

$$
\omega_{x}=2 \sqrt{\frac{\int_{-\infty}^{+\infty} \int_{-\infty}^{+\infty} I(x, y)(x-\bar{x})^{2} d x d y}{\int_{-\infty}^{+\infty} \int_{-\infty}^{+\infty} I(x, y) d x d y}} \text { where } \bar{x}=\frac{\int_{-\infty}^{+\infty} \int_{-\infty}^{+\infty} I(x, y) x d x d y}{\int_{-\infty}^{+\infty} \int_{-\infty}^{+\infty} I(x, y) d x d y}
$$

is the centroid of the beam profile in the $\mathrm{x}$-direction.

For Gaussian beams, the D4 $\sigma$ method gives the same result as the $1 / \mathrm{e}^{2}$ method, whereas for other beam

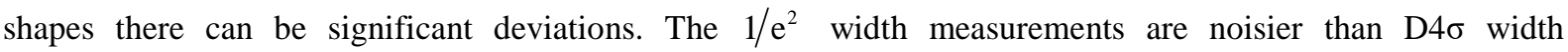
measurements depending on the integral of all the pixels contained in each frame. Let us add that for multimodal marginal distributions (a beam profile with multiple peaks), the D4 $\sigma$ width would be a better choice.

To calculate energy inside the aperture in the Z-scan configuration we must integrate over a radius $r_{a}=\omega_{i}\{0.5 \log [1 /(1-S)]\}^{1 / 2}$, where $S$ is the closed aperture linear transmittance and $\omega_{i}$ is the beam waist in the 
image plane in the linear regime. The latter is physically equal to the beam waist at the entry $\left(\omega_{i}=\omega_{e}\right)$ because the magnification of the considered $4 \mathrm{f}$ system is equal to 1 .

a)

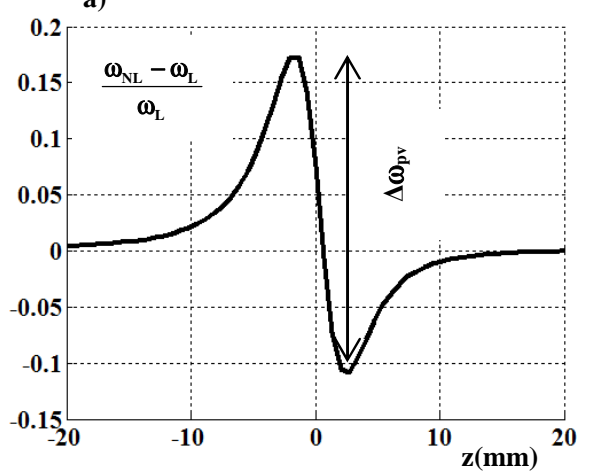

b)

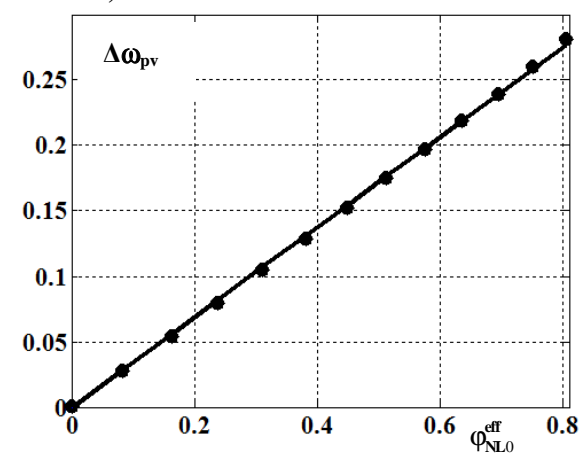

Figure 2: (a) Relative variation of the beam waist versus $z$. (b) Calculated $\Delta \omega_{p v}$, versus the effective phase shift at the focus for Gaussian input beam showing a linear dependence.

Fig. 2 (a) shows the simulation results of $\left(\omega_{\mathrm{NL}}-\omega_{\mathrm{L}}\right) / \omega_{\mathrm{L}}$, the relative variation of the beam waist versus $\mathrm{z}$, the position of the sample in the focus, where $\omega_{\mathrm{NL}}$ and $\omega_{\mathrm{L}}$ denote respectively the mean values (measured along $\mathrm{x}$ and $y$ ) of the beam waist defined in (2) of the NL profile and the linear one at the output of the 4f system. The other parameters are $\varphi_{\mathrm{NL} 0}^{\text {eff }}=0.8$ and $\mathrm{q}_{0}=0.58$. The difference between the peak and the valley $\Delta \omega_{\mathrm{pv}}$ shown in Fig. ${ }^{\circ}$ (b), is a function of the effective phase shift at the focus for a Gaussian input beam showing a linear relation $\left(\Delta \omega_{\mathrm{pv}}=0.34 \times \varphi_{\mathrm{NL} 0}^{\text {eff }}\right)$. Note that this formula allows to measure $\mathrm{n}_{2}$ when the other parameters in $\varphi_{\mathrm{NL} 0}^{\text {eff }}$ are known. Moreover the linearity remains valid in presence of relatively high NL absorption (here $\mathrm{q}_{0}=0.58$ ).

\section{EXPERIMENTAL RESULTS}

The detailed description of our experimental setup is given in [10]. Excitation is provided by a Nd:YAG laser delivering linealy polarized $17 \mathrm{ps}$ single pulses at $\lambda=1064 \mu \mathrm{m}$ with $10 \mathrm{~Hz}$ repetition rate. The input intensity is varied using a half-wave plate and a Glan prism. A beam splitter at the entry of the setup (Fig. 1) allows to monitor any fluctuation occurring in the incident laser beam. The focal lengths of lens $L_{1}$ and $L_{2}$ are equal to 20 $\mathrm{cm}$. To detect the light in the image plane we use a $1000 \times 1018$ pixels cooled $\left(-30^{\circ} \mathrm{C}\right) \mathrm{CCD}$ camera $\left(12 \times 12 \mu \mathrm{m}^{2}\right)$. Open and closed aperture signals are numerically processed from the acquired images. The CCD camera allows performing Z-scan, Baryscan and D4 $\sigma$ profiles out from the same acquired images at each z-position by numerically changing the soft aperture. Two sets of acquisitions are performed in the linear and the NL regimes in order to correct for the diffraction effects due to sample inhomogeneities and imperfections by subtracting the data related to the scans in each regime. When using the D $4 \sigma$ method one have to calculate the centroid of the beam before processing the beam waist using eq. 2 . This processing inside the images is equivalent to follow the pointing instability of the pulsed laser and thus reduces the noise that could appear with hard apertures as the ones used with Z-scan and Baryscan. Fig. 3 (a) shows the experimental results for $\mathrm{As}_{2} \mathrm{Se}_{3}$, a chalcogenide glass with relatively high NL absorption. The D $4 \sigma$ results (filled squares) can be compared with those of Baryscan (circles) using pulsed laser beams. Notice that the useful Baryscan signal is totally buried inside the noise due to laser pointing instability while the beam waist measurement method gives a cleaner signal with a higher signal to noise ratio. The same behaviour could be found for Z-scan using hard aperture. The calculated profiles (solid line for D $4 \sigma$ and dashed line for Baryscan) are shown in the same figure. The agreement is very good. The NL parameters obtained were: $n_{2}=(2.1 \pm 0.34) \times 10^{-18} \mathrm{~m}^{2} / \mathrm{W}, \varphi_{\mathrm{NL} 0}^{\text {eff }}=0.26$, and $\beta=2.2 \pm 0.2 \mathrm{~cm} / \mathrm{GW}$ (see the inset in Fig. $3 \mathrm{~b}$ ). Other parameters are: $\mathrm{I}_{0}=4.4 \mathrm{GW} / \mathrm{cm}^{2}$ (absolute measurement obtained as in [11]), $\alpha=0$, $\lambda=1.064 \mu \mathrm{m}, \omega_{\mathrm{i}}=1.9 \mathrm{~mm}$ and $\mathrm{L}=0.64 \mathrm{~mm}$. Moreover the $\mathrm{D} 4 \sigma$ method is unambiguously two times more sensitive than the Baryscan method. Physically we consider the totality of the pixels in order to measure the beam waist. So the quantity of information is twice that obtained with the razor blade in Baryscan. This is illustrated in Fig. 3 (a) by the difference between the peaks and the valleys inside the two scans showing approximately a factor 2 in the sensitivity. Fig. ${ }^{\circ} 3$ (b) shows the comparison of the beam waist relative variation (squares) and the usual normalized Z-scan transmittance (stars) which is the result of the division between the closed and the open aperture Z-scan traces shown in the inset. The radius of the soft circular closed aperture is calculated for $\mathrm{S}=0.73$ to optimize the signal to noise ratio [5]. The NL absorption profile is obtained by integrating over all the pixels. To obtain a good comparison the centroid method is used for both Z-scan profiles in order to monitor the beam transversally for pointing laser fluctuations (Fig. 3b). The calculations have been 
made using the same acquired images showing unambiguously approximately the same sensitivity. However, the advantage in the $\mathrm{D} 4 \sigma$ method is that there is no need to divide two different $\mathrm{Z}$-scan traces. The direct measurement of the beam waist gives directly the signal of the NL refraction.

a)

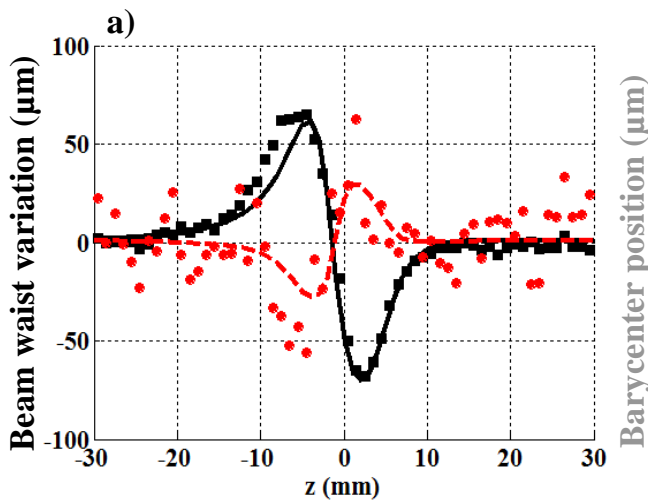

b)

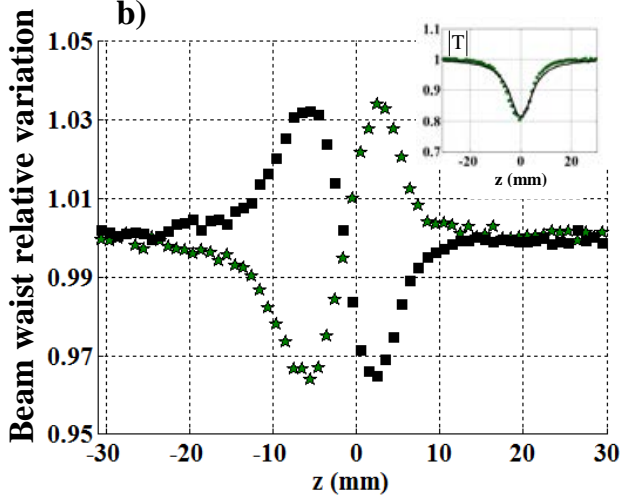

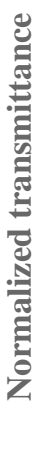

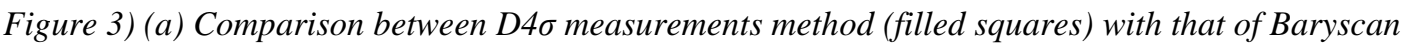
(circles). The solid and dashed lines show the numerical simulations. (b): Comparison of the beam waist relative variation (squares; vertically shifted to 1) and the usual normalized Z-scan transmittance (stars) (division of the closed and the open aperture Z-scan traces) for highly NLA material. The inset shows the open aperture Z-scan transmittance. The processing has been made using the same acquired images.

Generally, the hard aperture at the output of an experimental setup intercepts the laser profile at different position due to the laser pointing instability. This leads to a variation of the transmitted intensity showing a fluctuating noise. The use of the CCD camera allows to follow the centroid of the image. Then, it is possible to apply a numerical soft apertures of different shapes. For arbitrary (possibly not Gaussian) beam, it is recommended to use the D4 $\sigma$ or second moment beam width which is the ISO standard definition [12]. To improve the signal-to-noise ratio, the centroid of the beam must be calculated for the Z-scan traces as well as in the Baryscan method.

\section{CONCLUSION}

The $\mathrm{D} 4 \sigma$ method consisting of measuring the beam radius at the output of a $4 \mathrm{f}-\mathrm{Z}$-scan method using a CCD camera provides a signal having a peak-valley shape. One of the advantages of this technique is to be insensitive to pointing instability of the pulsed laser because no hard aperture is employed as in the usual Z-scan or Baryscan methods. Numerical calculations allow to obtain simple relations that can be used for the measurements simplifying the procedure when a NL absorbing material is used. Moreover the normalization process does not lead to any ambiguity especially when the intensity of diffracted light is very low.

\section{REFERENCES}

[1] M. Sheik-Bahae, et al., Sensitive measurement of optical nonlinearities using a single beam, IEEE J. Quant. Elect. 26, 760, (1990)

[2] T. Godin, M et al., Baryscan: a sensitive and user-friendly alternative to Z-scan for weak nonlinearities measurements, Opt. Lett. 36, 8, (2011)

[3] M. R. Ferdinandus, et al.,Dual-arm Z-scan technique to extract dilute solute nonlinearities from solution measurements, Opt. Mat. Express 2, 12, (2012)

[4] K. Fedus and G. Boudebs, Sensitivity of the $4 \mathrm{f}$ coherent imaging system used in degenerate multi-wave mixing experiments, J. Opt. Soc. Am. B, 26, 2, 244, (2009).

[5] K. Fedus, G. Boudebs, Experimental techniques using $4 \mathrm{f}$ coherent imaging system for measuring nonlinear refraction, Optics Communications 292, 140, (2013).

[6] P. Chen, et al., Two-dimensional Z scan for arbitrary beam shape and sample thickness, J. Appl. Phys. 85, 7043, (1999)

[7] G. Tsigaridas, et al., V. Giannetas, Z-scan technique through beam radius measurements, Appl. Phys. B 76, 83, (2003)

[8] G. Boudebs, et al., Third-order susceptibility measurements by nonlinear image processing J. Opt. Soc. Am. B, 13, 145013, (1996),

[9] G. Boudebs, et al., Contrast increasing by third-order nonlinear image processing: a numerical study for microscopic rectangular objects, Optics Communications, 150, 287, (1998).

[10] K. Fedus, G. Boudebs, and H. Leblond, Degenerate multi-wave mixing inside a $4 \mathrm{f}$ imaging system in presence of nonlinear absorption, Applied Physics B-Lasers and Optics, 100, 4, 827, (2010).

[11] G. Boudebs, K.Fedus, Absolute measurement of the nonlinear refractive indices of reference materials, J. Appl. Phys. 105, 103106, (2009)

[12] ISO Standard 11146, Lasers and laser-related equipment - Test methods for laser beam widths, divergence angles and beam propagation ratios, (2005), http://www.iso.org/iso/catalogue_detail.htm?csnumber=33626, 